\title{
“Consumocracia”. El consumo político como forma de participación de la ciudadanía
}

\author{
Amparo Novo VÁzQUEZ \\ Universidad de Oviedo \\ anovo@uniovi.es
}

Recibido: 08-06-2013

Aceptado: 26-02-2014

\section{Resumen}

El consumo político o political consumerism es una forma de participación en la que la ciudadanía utiliza el mercado para expresar sus preocupaciones políticas. Realizar la compra de un producto (buycott) o no (boycott) es una decisión personal justificada, basada en valoraciones éticas, medioambientales o políticas que se hacen de los procedimientos realizados por las empresas o los gobiernos. Las nuevas tecnologías y los distintos medios de difusión informativa influyen en el alcance de este tipo de movilización política dada la importancia creciente de las redes sociales transnacionales y las comunidades en línea, las cuales contribuyen a la formación de opinión de la ciudadanía y a su participación en un nuevo espacio "sub-político". De tal forma que interesa tanto el consumidor crítico o consciente que convierte su hábito de compra en hábito político como el contexto a través del que obtiene la información, y los distintos actores -los activistas o movimientos sociales- con los que participa, convirtiendo el mercado en arena pública y objeto de reivindicaciones para la práctica de la democracia. Este artículo tiene como objetivos: conceptualizar la participación del consumidor desde la perspectiva de las teorías sociológicas de la modernidad, presentar algunas de las metodologías utilizadas para medir y evaluar la influencia de los consumidores políticos y ver en qué medida su empoderamiento a través del mercado podría acercarse a una "consumocracia".

Palabras clave consumocracia, consumo político, acción colectiva individualizada, participación política y ciudadanía

\section{“Consumecracy”. Political Consumerism as a way of citizen participation}

\begin{abstract}
Political consumerism is a form of participation in which citizens use the market to express their political concerns. Making the purchase of a product (buycot) or not (boycott) is a justified personal decision based on ethical values, environmental or political concerning the procedures performed by companies or governments. New technologies and different information media affect the scope of this type of political mobilization given the growing importance of transnational social networks and online communities, which contribute to the formation of the public opinion and to the participation in a new "sub-political" space. So we are interested in: a) critical or conscious consumers transforming their purchase habit in political habit, and b) the context through which they obtain the information, and the different actors, activists and social movements with which they participate, transforming the market into a public arena and a subject of claims for the practice of democracy. This article therefore
\end{abstract}


aims to: conceptualize consumer involvement from the perspective of sociological theories of modernity, present some of the methodologies used to measure and evaluate the influence of political consumers, and see to what extent their empowerment trough the market might approach a "consumecracy".

Key words: consumecracy, political consumerism, individualized collective action, political participation, citizenship

\section{Referencia normalizada}

Novo Vázquez, A. (2014). “““Consumocracia”. El consumo político como forma de participación de la ciudadanía”. Política y Sociedad, Vol.51 Núm. 1 121-146

Sumario: Introducción. 1.Conceptos y teorías del consumo político. 2.El campo del consumo político: repertorios de participación y actores. 3.El estudio del consumo político: encuestas internacionales y nacionales. 4.Consumidores políticos y estilos de vida. 5. "Cosumocracia” o el empoderamiento de los consumidores ante el mercado 


\section{Introducción}

Como resultado de los cambios trascendentales del sistema capitalista y, en particular, de la globalización, en los últimos tiempos los límites de la esfera política tienden a diluirse, con el surgimiento de nuevos actores como grupos de interés, movimientos sociales transnacionales, redes sociales online, así como de nuevos repertorios de acción que dan lugar a una "reinvención del activismo político" (Norris, 2002: 6). De esta manera surgen nuevas formas de participación, como el voluntariado, que se convierten en formas alternativas de participación política (Putnam, 2000; Norris, 2001). Además, si a esto unimos el alcance de Internet (van Laer y van Aeslt, 2009) tanto para apoyar los viejos repertorios de acción (envío de correo electrónico a un representante político) como para acciones que solo se pueden realizar vía web (plataformas para promover el cambio social como Change.org) se puede determinar que estamos ante un nuevo contexto social global en el que la ciudadanía utiliza nuevas vías para ejercer sus derechos de implicación política en las democracias representativas.

De las distintas alternativas de participación ciudadana se va a estudiar el consumo político o political consumerism. La ciudadanía a través del mercado elige a productores y productos en función del bienestar individual o familiar o de las valoraciones éticas y políticas que se hagan de los procedimientos realizados por las empresas y los gobiernos (Andersen y Tobiasen, 2001; Micheletti, 2002; 2003; Micheletti, Follesdal y Stolle, 2003). Diversos estudios apuntan la importancia de los factores contextuales, como la globalización, la economía, las instituciones políticas y la cultura para explicar el consumo político (Beck, 2001; Micheletti y Stolle, 2005; Neilson y Paxton, 2010; Koss, 2012).

En este trabajo se pretende dar respuesta a preguntas tales como de qué manera se puede conceptualizar la participación de los consumidores conectando los paradigmas de Giddens y Beck. También se quiere saber de qué forma puede influir su participación en los comportamientos de otros actores políticos como las multinacionales o los gobiernos. Cómo puede ser medida y evaluada la influencia de los consumidores políticos o qué factores se asocian con el éxito de su participación. En definitiva se va a comentar qué significa el consumo político, qué se ha hecho hasta el momento y cuáles son los principales horizontes de análisis. Es preciso apuntar que hasta la fecha no se ha realizado una sistematización relativa a las principales metodologías de esta forma de participación.

En las líneas siguientes se va a exponer en primer lugar lo que se entiende por consumo político y algunos de los conceptos vinculados a esta forma de acción política, asimismo se hará una descripción de las teorías y cuáles tomamos como referencia para enmarcar este nuevo tipo de reivindicación. En segundo lugar, las formas de consumo político propuestas por Micheletti y Stolle (2004) se relacionarán con dos tipologías actuales: una elaborada por Ekman y Anna (2009) de repertorios de participación política con la intención de ver de manera más gráfica los diferentes contextos de actuación; y otra la tipología de van Laer y van Aeslt (2009) de acción colectiva online de los movimientos sociales. También se realizará una 
breve descripción de quienes son los principales actores involucrados en este tipo de participación política. En tercer lugar, se expondrán las principales encuestas internacionales y nacionales realizadas hasta el momento, para ello se ha seleccionado además de otras investigaciones aquellas que citan el artículo de mayor repercusión de Dietlind Stolle, Marc Hooghe y Michelle Micheletti (2005) "Politics in the Supermarket: Politics Consumerism as a Form of a Political Participation”. En esta misma sección se presentan algunas de las investigaciones que han utilizado metodología cualitativa con el objetivo de conocer cuáles son las variables independientes que determinan el consumo político. En un cuarto apartado se indicarán los perfiles de los consumidores políticos y cuáles son sus estilos de vida. Y, para finalizar, se realizarán unas reflexiones que nos permitan definir el concepto de “consumocracia”.

\section{Conceptos y teorías del consumo político}

Realizar la compra de una mercancía (buycott) o no (boycott) es una decisión personal justificada a la que Micheletti (2003, 2010: 25-29) ha denominado “acción colectiva individualizada”. Es decir, los ciudadanos responsables construyen espacios cotidianos de acción individual o colectiva para hacer frente a problemas que afectan al bienestar común. Al sentirse alejados de las formas tradicionales de participación, los responsables políticos no son vistos como capaces de percibir los riesgos creados por las empresas y en última medida por determinadas políticas públicas, de tal forma que crean un espacio diferente y alternativas de participación.

Ante este nuevo contexto social y político las teorías sociológicas de la modernidad vinculan la globalización con la política de la vida cotidiana (Siim, 2003: 45), de tal forma que las personas se ven inmersas en un proceso de "desvinculación y revinculación hacia nuevas formas de vida en la sociedad industrial en sustitución de las antiguas, en donde de manera individual tienen que producir, representar y continuar sus propias biografías” (Beck et al 2001: 28).

Se produce, en palabras de Beck et al (2001 [1997]) el paso de una sociedad moderna a una reflexiva cuyo escenario de participación se expresa ahora fuera de la esfera tradicional. En el marco de la teoría de la modernización reflexiva acuña el concepto de "sub-política" que hace referencia a la vía alternativa de intervención pública de personas, actores sociales y colectivos de hacer política, con la intención de influir en la "autoorganización de lo político" (Ibid: 33). Ahora, "los consumidores son actores sub-políticos en el sentido de que perciben la política como un sistema de gobernanza (más que en el sentido de gobierno) en donde ellos mismos juegan un papel activo y asumen una responsabilidad para hacer frente al riesgo de una manera reflexiva” (Tobiasen, 2004: 142). Esto puede considerarse el paso del gobierno a la gobernanza (Todt y González, 2006).

Por otro lado, Siim (2003: 4-5) considera que los paradigmas de Giddens y Beck son interesantes desde la perspectiva de género ya que ambos hablan de una nueva dimensión de la política que al menos potencialmente conecta con temas de la vida 
cotidiana así como la participación de la ciudadanía fuera de las clásicas instituciones políticas (Beck, Giddens y Lasch, 2001 [1997]). En este nuevo espacio en el que se usa el mercado para transmitir las preocupaciones éticas, medioambientales o políticas, las mujeres, las personas jóvenes y las de mayor nivel educativo, como se verá más adelante, tienen una presencia importante. Por este motivo parece interesante la aportación desde la perspectiva de la teoría feminista del concepto de Iris Marion Young (1990) de la "política de la diferencia" o la transformación de la política "desde abajo" ya que sirve de referencia para enmarcar también teóricamente el consumo político. Los diferentes estudios realizados hasta el momento dan cuenta de que es una forma de participación realizada en algunos países mayoritariamente por mujeres y en otros, sus acciones tanto individuales como colectivas (asociaciones voluntarias, cooperativas o movimientos sociales) son más que significativas en comparación con otras formas de acción política más tradicionales. En muchas ocasiones las mujeres han hecho uso del poder del consumo para cambiar la sociedad (Young, 1994).

Pero si bien estructuramos nuestro argumento bajo el paraguas de ambas teorías no podemos obviar que también desde la teoría del capital social se ha analizado este tipo de activismo. En este caso parten del supuesto de que si las personas consumidoras son miembros de asociaciones voluntarias esto les provee de competencias para desenvolverse en el entorno de la acción colectiva (Hooghe y Stolle, 2003; Putnam 1993, 2000), así se puede decir que pertenecer a redes facilita ser consumidor político. A este respecto, suscribimos la crítica a este marco teórico basada en que en la mayoría de las sociedades occidentales el incremento del nivel educativo de las mujeres (a veces superior al de los hombres) y su incorporación al mercado laboral tiene un "efecto positivo en la pertenencia a asociaciones voluntarias y en el interés político, sin embargo, la mayor participación de las mujeres rara vez es reconocido en la literatura sobre el capital social”, a causa de una orientación unilateral hacia las estructuras formales de participación (Micheletti y Stolle, 2003: 2).

El proceso de modernización y los valores postmaterialistas como la preocupación por el medioambiente, la búsqueda de la igualdad, la inclusión de las minorías, el respeto a los derechos humanos o el desarrollo sostenible, preocupa a una mayoría considerable que plantea demandas acordes con los nuevos valores, motivo éste por el que surgen nuevos espacios de acción política en donde expresarse (Inglehart, 1997). El postmaterialismo y el sentimiento de obligación moral con el medioambiente o los derechos humanos está muy próximo a lo que Bennett (1998) denominó "lifestyle politics" o política del estilo de vida (Shah et al 2007: 219). 


\section{El campo del consumo político: repertorios de participación y actores}

\subsection{Tipologías de repertorios de participación}

La expansión de las actividades consideradas como participación política se produce en los años noventa. A partir de entonces se realizan importantes estudios que avanzan nuevas tipologías. Así entre otros, se pueden destacar el de Parry et al. (1992) que comprueban el carácter multidimensional de la participación política y descubren alternativas de acción que se resumen en seis grandes tipos: voto, campaña de partidos, acción colectiva, contactos, acción directa y violencia política. También Verba et al. (1995: 72) presenta una tipología en la que reducen la participación política a cuatro actividades principales: voto, campaña, contacto con los funcionarios públicos y actividades comunitarias o cooperativas.

En la actualidad, las investigaciones se dirigen no sólo al estudio de la participación política sino también a otras formas en las que se incluyen las actividades que se refieren al compromiso cívico. A este respecto es interesante considerar la "nueva tipología” de Ekman y Amna (2009, 2012). Según estos autores, la distinción entre formas de participación, latentes y manifiestas es fundamental, además hay que añadir que pueden ser realizadas individualmente o colectivamente. Como se puede observar en la tabla 1, dentro de las formas de participación cívica (política "latente") se encontraría la implicación social (atención) -interés en la política, pertenencia a un grupo con fines sociales o identificación con una ideología o partido- y el compromiso cívico (acción) -discutir temas sociales o políticos con amigos o a través de Internet, actividades con organizaciones de base comunitaria o leer periódicos y ver en la televisión programas sobre temas políticos-. Por lo que respecta a la participación política "manifiesta" se encontrarían la participación política formal (voto, actividad en un partido, un sindicato o contactar con representantes políticos o civiles) y la participación política extraparlamentaria a través de acciones legales (consumo político, firma de peticiones o participación en protestas) o acciones ilegales (ocupación de edificios, ataques contra la propiedad o desobediencia civil).

Según esta clasificación, la acción individualizada que realizan los consumidores se enmarca en lo que denominan participación política "manifiesta", extraparlamentaria, realizada a través de acciones legales: a través del buycott (consumo positivo), cuando se dejan guiar por los sistemas de etiquetado como puede ser la compra de productos de comercio justo, y del boycott (consumo negativo) o no comprar ciertos bienes por razones éticas, medioambientales o políticas. De manera colectiva, se puede realizar a través de la participación en nuevos movimientos sociales o participación en protestas o huelgas. 
Tabla 1. Tipología de repertorios de participación política

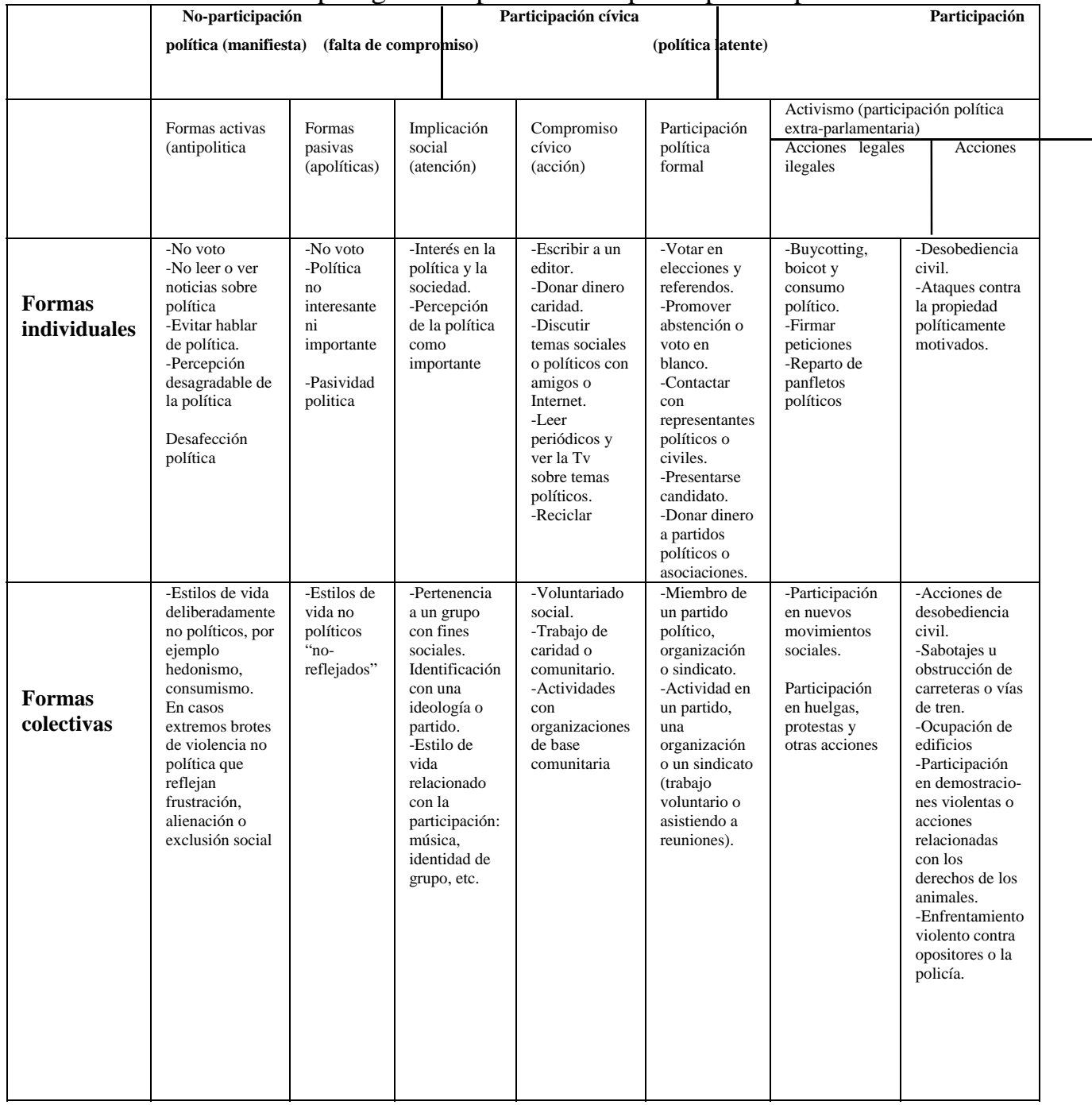

Fuente: Enkma y Anna (2009)

La última década se ha caracterizado por la presencia y el papel de Internet en la vida de una mayoría significativa de personas en prácticamente todas las sociedades. La expansión revolucionaria de las tecnologías de la información y comunicación ha puesto a nuestra disposición nuevos recursos. Así, a través del uso de los emails, blogs u otros sitios webs de publicación, un número creciente de personas está utilizando la red difundiendo información, interactuando e involucrando a diferentes públicos, ahora en un nuevo contexto, el "online”. Los principales actores en este ámbito son las personas jóvenes que se desvinculan de las formas tradicionales 
de la política invirtiendo su tiempo en otras formas de participación más dinámicas en función de intereses transitorios.

Ahora se presenta, de manera resumida, la tipología elaborada por van Laer y van Aeslt (2009: 5-18) de los "repertorios online” de acción colectiva de los movimientos sociales, con la intención de ver de qué manera las personas pueden participar y de conocer los nuevos espacios de acción de los consumidores políticos. Esta clasificación cuenta con dos dimensiones como se puede observar en la figura 1:

1. Acciones que se apoyan en Internet versus acciones que solo se pueden realizar a través de Internet. Hace una diferenciación entre "viejos" repertorios que se apoyan en Internet (envío de correo electrónico a partidos políticos o a miembros de un gobierno, o a una empresa) y "nuevos” tipos de acción basados en Internet como pueden ser un email bomba o una petición online.

2. Umbrales bajos en comparación con umbrales altos. Algunas formas de acción conllevan más riesgo y un mayor coste o compromiso que otras. Dentro de los umbrales bajos estaría la firma de peticiones, las manifestaciones legales, y, dentro de los umbrales altos, las manifestaciones ilegales o las acciones violentas.

A continuación se enumeran distintas posibilidades de acción para ello se estructuran en 4 cuadrantes teniendo en cuenta las dos dimensiones señaladas con anterioridad:

$1^{\circ}$ cuadrante: acciones apoyadas en Internet con umbral bajo. Donar dinero, ser consumidor activo consciente (por ejemplo, eBay, Would of Good Development), participar en manifestaciones legales a nivel transnacional.

$2^{\circ}$ cuadrante: acciones apoyadas en Internet con umbral alto. Manifestaciones trasnacionales, la participación en foros transnacionales (Foro Social Mundial o el Foro Europeo), el bloqueo y ocupación de calles o la destrucción de la propiedad.

$3^{\circ}$ cuadrante: acciones basadas en Internet con umbrales bajos de participación. Peticiones en línea (Facebok, Actuable.com o Change. com...), emails bomba/sentadas virtuales; envíos masivos a un Ministerio o dirección general; recepción de información masiva de un sitio web de tal forma que el servidor no pueda hacer frente.

$4^{\circ}$ cuadrante: acciones basadas en Internet con umbrales altos. Sitios web protesta, medios de comunicación alternativos, interferencia cultural o culture Hamming (por ejemplo, alteración de logos corporativos) y hacktivismo (denegación del servicio (DoS), ciberterrorismo). 
Figura 1. Repertorios de acción social online de los movimientos sociales

\begin{tabular}{|c|c|c|c|}
\hline $\begin{array}{l}\text { Apoyado en } \\
\text { Internet }\end{array}$ & en & & $\begin{array}{l}\text { Basado en } \\
\text { Internet }\end{array}$ \\
\hline $\begin{array}{l}\text { Umbral } \\
\text { alto }\end{array}$ & $\begin{array}{l}\text { Acciones más violentas/ } \\
\text { Destrucción de la propiedad } \\
\text { Sentada/ocupación } \\
\text { Manifestación transnacional/encuentro }\end{array}$ & $\begin{array}{l}\text { Culture jamm } \\
\text { Sito web }\end{array}$ & Hacktivismo \\
\hline $\begin{array}{l}\text { Umbral } \\
\text { bajo }\end{array}$ & $\begin{array}{l}\text { Manifestación legal } \\
\text { Comportamiento consumidor } \\
\text { Donación dinero }\end{array}$ & Petición online & $\begin{array}{l}\text { Email bomba/sentada virtual } \\
\qquad 3\end{array}$ \\
\hline
\end{tabular}

Fuente: Elaboración a partir del cuadro realizado por van Laer y van Aeslt (2009: 5)

Internet ofrece ventajas a la hora de difundir información, no sólo facilita la conexión entre productores y compradores conscientes, sino también posibilita la comunicación o la transmisión de campañas tanto de boicot como de buycott iniciadas por los movimientos sociales.

Y para finalizar esta sección es imprescindible señalar la clasificación elaborada por Micheletti, Stolle y Berlin (2012: 146) en donde identifican cuatro formas de participación: 1) boycotts o no comprar un producto o marca de manera consciente; 2) buycotts o comprar un producto o marca tomando como referencia la etiqueta; 3) las acciones discursivas o valoraciones críticas sobre las prácticas corporativas o 
difusión de opiniones sobre consumo, y 4) elección del estilo de vida o las decisiones personales basadas en valores sobre cómo orientar los propios recursos.

\subsection{Actores involucrados en los contextos de participación}

En los diferentes contextos de participación se ven implicados diversos actores políticos que interesa identificar y saber cuáles son los posibles efectos de sus comportamientos. Por un lado, el consumidor consciente que de forma individual expresa sus valoraciones a través del mercado. Sus acciones pueden ser medidas a través de encuestas, de este modo se puede observar esta nueva forma de compromiso cívico (Kelley, 1899/2008; Hertz, 2001; McGregor, 2002) y político. En este caso, los actores políticos tradicionales, partidos o gobiernos, hacen uso de ellas percibiendo las demandas de la población que, en algún caso, se integrarán en las agendas institucionales.

Por otro lado, los activistas, actores políticos imprescindibles que a través de la cooperación colectiva promueven reivindicaciones como la desaprobación ante determinadas prácticas ética o políticamente cuestionables efectuadas por corporaciones, empresas o gobiernos (Clarke et al, 2007). Así, los activistas canalizan de esta forma los conflictos de intereses entre los distintos agentes involucrados en el mercado de producción, distribución y consumo de alimentos con el fin de llegar a establecer las agendas públicas tanto de los consumidores como de los gobiernos. En este terreno los medios de comunicación juegan un papel fundamental transmitiendo a la opinión pública las quejas formuladas, lo que sin duda puede afectar tanto económicamente a las empresas como poniendo en cuestión las políticas de los gobiernos.

Por tanto, la acción conjunta de ambos actores políticos (consumidores conscientes y activistas) puede llegar a alterar las agendas políticas de los gobiernos a nivel local, nacional, regional y mundial; de este modo "no es una exageración afirmar que los grupos de iniciativas ciudadanas han adquirido poder político" (Beck et al, 2001: 33-34).

Realizar la compra en el mercado y recibir el ticket puede ser interpretado simbólicamente como el acto de votar, de tal forma que a través del "voto" de los consumidores en las cajas, las empresas sienten que tienen que rendir cuentas (Jacobsen y Dulsrud, 2007; Johnston, 2008). Así "en el momento en que se usa el mercado como forma de acción política, la empresa ha de actuar en consecuencia y asumir el papel de actor político. Las razones de que las empresas se conviertan en actores políticos son: en primer lugar, el hecho de que las decisiones y acciones que ejecutan influyen irremediablemente en la realidad social, económica, política, cultural o medioambiental; en segundo lugar, cada vez más se requiere de su participación y colaboración en la búsqueda de soluciones a problemas políticos; y, por último, son actores políticos ya que se espera que tengan una participación en la responsabilidad social corporativa” Rask Jensen (2001: 5). 
La importancia política de las corporaciones se puede medir "por un lado, porque tienen efectos sobre las condiciones de vida de las personas, desde la creación de empleos y la reducción de la degradación del medio ambiente, estas son consecuencias de una sub-política pasiva ya que su significado se construye desde afuera, pero también es activa a través de las diversas formas en que las empresas pueden tratar de influir en el proceso político formal a través de los grupos de presión, por ejemplo. También implica la interacción de su negocio con el entorno social más amplio ya que las empresas buscan legitimar sus acciones” (Holzer y Sorensen, 2003: 87). Así, a través de las prácticas de responsabilidad social corporativa (RSC) las empresas reinvierten parte de sus beneficios en el desarrollo de aspectos humanos, sociales y medioambientales (Blanco, 2006). La absorción parcial de las responsabilidades sociales por las empresas implica una percepción más débil del poder del Estado frente a las grandes corporaciones y empresas. Los recursos financieros invertidos por éstas en programas de desarrollo comunitario implican una nueva forma de activación de la ciudadanía (Echegaray, 2010).

En definitiva, el impacto de las acciones de los consumidores, las corporaciones, movimientos sociales, empresas y asociaciones se refleja en la agenda política de los gobiernos lo que hace pensar en un cambio en el funcionamiento del sistema político ahora más global, en donde los diferentes actores involucrados juegan un papel importante de colaboración, dando lugar a una gobernanza orientada hacia objetivos específicos.

\section{El estudio del consumo político: encuestas internacionales y nacionales}

Es preciso señalar que el consumo político no es un fenómeno nuevo; los boycotts se remontan al siglo XIX y eran los movimientos sociales los principales agentes de difusión de las campañas. Existen diferencias con los "nuevos movimientos sociales" que se basan en la "identidad política" de personas que se movilizan buscando objetivos diferentes en torno a valores personales relacionados con estilos de vida (Bennet, 2012); en este caso el objetivo es empoderar a la sociedad frente al mercado. A lo largo del tiempo, los movimientos sociales han tratado de concienciar a los consumidores en asuntos como el respeto a los derechos humanos, la difusión del comercio justo, el consumo sostenible o el cumplimiento de las diferentes normativas (Dubuisson-Quellier, 2010: 1865). De esta forma se ha ido expandiendo la conceptualización del consumo político desde los primeros estudios sobre boycotts (Inglehart, 1997: 313, Andersen y Tobiasen 2003, Norris 2002: 198) o buycotts comprar productos en atención a la información que aparece en el etiquetado, si son productos ecológicos o de comercio justo- (Micheletti, 2003).

En los años setenta se realizan los primeros estudios sobre consumidores conscientes, término utilizado para definir a aquellas personas socialmente responsables que entre sus metas está el lograr cambios (Webster, 1975). A partir de entonces nociones como consumo ético, consumo social consciente o consumo sostenible entre otros forman un concepto ampliado según el cual la ciudadanía se convierte en 
consumida crítica y consecuentemente en agente de cambio social (Micheletti, 2003). No es suficiente comprar o no un producto, el consumidor toma sus decisiones bien siguiendo una campaña ética, política o medioambiental determinada, bien consultando la información que aparece en las etiquetas de los productos que desea comprar e incluso no acudiendo a un establecimiento por razones fundamentadas. Es preciso señalar que entre las diferentes motivaciones, las relacionadas con el nacionalismo cobran importancia en esta forma de participación política. Este es el caso de no comprar o comprar un producto porque procede de determinado país o de una Comunidad Autónoma.

El consumo político por tanto se convierte en una forma de participación política cada vez más importante en la práctica ciudadana de las democracias occidentales (Inglehart 1997: 313; Norris 2002; Boström et al. 2005; Dalton 2008; Strømsnes 2009), y de forma especial, en los países escandinavos. Es por ello que las investigaciones más completas han sido las elaboradas por científicas y científicos sociales de estos países, además de ser los precursores como ya se ha visto en su conceptualización (Micheletti, 2003; Boströn, 2003 y Klintman y Böstrom, 2004). Sin embargo, es necesario señalar que los estudios sobre la acción colectiva individualizada (boycott y buycott) y sobre la acción colectiva (movimientos sociales) en torno al consumo político aún son escasos tanto a nivel internacional como en España.

Para analizar el consumo político hay que tener en cuenta tres condiciones (Stolle, Hoghe y Micheletti, 2004: 254-255): el comportamiento (comprar o no ciertos productos); la sensibilización y motivación de los consumidores (ética, política o medioambiental) y la frecuencia y hábito (pauta de comportamiento).

En este trabajo, se pretende describir algunas de las investigaciones más sobresalientes, tanto cuantitativas como cualitativas, con la intención de observar cómo ha evolucionado esta nueva forma de participación, su repercusión y cómo se ha estudiado hasta este momento.

En el estudio realizado por Barners y Kaase (1979) aparece por primera vez la pregunta sobre boycott (Michelletti y Stolle 2012: 146). Las investigaciones de Andersen y Tobiasen (2003) Inglehart (1997: 313); Norris (2002: 198) demuestran a través de evidencias empíricas que el boycott es una práctica que va en aumento (Micheletti, 2005:1). Entre febrero de 2002 y julio de 2005 se realiza un estudio panel en EEUU en el que se analiza la relación entre el consumo político y el uso de los medios de comunicación (Shah et al., 2007). También hay que destacar la encuesta nacional Citizenship, Involvement, Democracy (CID) realizada por el Democracy and Civil Society at Georgetown University (Howard, Gibson, y Stolle, 2005) de 1001 entrevistas cara a cara en EEUU (16 de mayo a 16 de julio) con una tasa de respuesta del $40 \%$.

Además se han realizado encuestas monográficas en Suecia: Swedish National Survey (SOM 2003) en el marco del proyecto Political Consumption: Politics in a New Era and Arena (Stolle y Micheletti, 2005) o Consumption and Societal Issues Survey realizada en Suecia en 2009 a 1053 personas, enviada por correo a unas 3000 personas con una tasa de respuesta de 35\% diseñada para estudiar: hábitos de consumo, participación política, ideal de ciudadanía, valoración de políticas me- 
dioambientales o confianza en las instituciones (Berlin, 2011). La encuesta llevada a cabo a la población danesa durante el verano de 2004 (Tobiasen, 2005) o la encuesta piloto a una muestra de 1015 estudiantes en Canadá, Bélgica y Suecia (Stolle, Hooghe y Micheletti, 2005). Y, para finalizar, el estudio panel nacional Cooperative Campaign Analysis Project (CCAP) de seis olas de diciembre de 2007 a noviembre de 2008 a una muestra de 1191 votantes registrados (Gotileb y Wells, 2012).

En el contexto online, es interesante destacar la encuesta realizada a 1215 jóvenes en el marco del proyecto de ámbito europeo llamado CivicWeb. Saber la naturaleza, características, producción y uso e interpretación de los sitios web cívicos y políticos era su objetivo basado en la producción, la naturaleza y características de los sitios web cívicos y políticos y los usos e interpretaciones de estos sitios por las personas jóvenes (Vaar y Vreese, 2011: 404).

De carácter más general incluyendo mayor número de países está el Eurobarómetro, la Encuesta Europea de Valores, y la Encuesta Mundial de Valores en las que solo se les pregunta por acciones de boicot. Hay que señalar que en la primera ola de la Encuesta Social Europea (ESE) realizada durante 2002-2003 se incluyeron las dos preguntas que permiten el estudio del consumo político; en las siguientes olas sólo se introduce la cuestión de boicot a productos o empresas.

Parece oportuno reseñar algunos resultados de la primera ola de la ESE para observar las acciones de compra o boicot en 21 países europeos durante el período de 2002-2003. En Suecia o Suiza más del 30\% de la población ha boicoteado en los últimos doce meses, en el caso de España sólo un 8\% afirma haberlo realizado. En el caso de la compra de productos Suecia, Suiza, Dinamarca y Finlandia más del 40\% de la población lo practica, en España es el 12\%. Como señala Ferrer (2010: 250) existen diferencias entre países en las dos pautas de comportamiento siendo más significativa en el caso de la compra.

A partir del año 2002 algunas de las encuestas elaboradas por el Centro de Investigaciones Sociológicas (CIS) relativas a temas de ciudadanía, representación o participación (ver tabla 1), introducen las preguntas de boicotear y comprar productos por cuestiones políticas, éticas o medioambientales; ya en 2006 se incluye un bloque de cuestiones a través de las que se puede estudiar el consumo como forma de participación política. Se ve un incremento importante de más de 15 puntos porcentuales, en algunos casos, en nueve años.

En España, el estudio Ciudadanía, Participación y Democracia, nº 2450 del CIS de 2002, se incluyen las dos preguntas. En ese momento sólo un 6\% decía que había boicoteado en los 12 últimos meses y comprar productos por razones éticas, políticas o medioambientales, lo habían realizado un $12 \%$ de la muestra.

En el año 2004 en el estudio n ${ }^{\circ} 2575$ Ciudadanía y participación un 13\% dice que lo había practicado durante el año anterior. En el 2005 en el estudio ${ }^{\circ} 2588$ Representación y Participación política en España aparecen ambas preguntas unidas y el porcentaje de españoles que responden que han realizado boycott o buycott se elevaba al 18\%. En este mismo año se realiza el estudio n ${ }^{\circ} 2606$ Globalización y relaciones internacionales y ante la pregunta de si ha boicoteado o com- 
prado ciertos productos por razones políticas, éticas o para favorecer el medio ambiente el 14 de la población manifiesta haberlo practicado.

En el estudio n ${ }^{\circ} 2632$ Ciudadanía y participación de 2006 se introduce por vez primera un bloque de preguntas específico sobre consumo político. Así, en el último año, el 14\% de la población española dice haber realizado boycott y un 18\% practicó buycott sin que haya diferencias apreciables por cuestión de sexo. Al ser preguntados por las causas que les llevaron a realizar el boycott un $49 \%$ respondió que sus motivos eran el no respeto al medioambiente y un 33\% porque se utilizaba el trabajo infantil. Por otro lado, los motivos del buycott fueron para el $65 \%$ porque respetaban el medio ambiente; el $35 \%$ por ser productos del tercer mundo (comercio justo, etc) y un 32\%, por apoyar a pequeños productores (cooperativas o pequeños agricultores). Un $72 \%$ de los españoles conocía la existencia en algunos establecimientos de productos de comercio justo, de hecho, el 26\% los había adquirido alguna vez. Por otro lado, el $46 \%$ recordaba alguna campaña de boicot a productos o empresas en España, más los hombres (54\%) que las mujeres (38\%); el 61\% citan al cava y el $35 \%$ productos y empresas catalanas, la diferencia entre hombres y mujeres no es significativa. El 84\% se enteraron a través de los medios de comunicación; el 77\% a través de la tv, radio 44\%, prensa escrita 38\% e internet el 13\%.

En el estudio $\mathrm{n}^{\circ} 2736$ de 2010 Internet y participación política se pregunta si en los últimos doce meses compró ciertos productos por razones políticas o para favorecer el medio ambiente, un $25 \%$ de la muestra afirma que lo ha realizado, un $27 \%$ de mujeres frente a un $24 \%$ de hombres; Boicoteó o dejar de comprar ciertos productos por razones políticas, éticas o para favorecer el medio ambiente lo han realizado un $20 \%$ de la muestra $21 \%$ de mujeres frente a un $19 \%$ de hombres.

En el estudio $\mathrm{n}^{\circ} 2860$ de 2011 sobre Preferencias sobre los procesos de toma de decisiones políticas se les preguntaba si habían boicoteado o dejado de comprar ciertos productos por razones políticas, éticas o para favorecer el medio ambiente un $17 \%$ del total de la muestra respondió afirmativamente el 18\% mujeres frente al $16 \%$ de los hombres.

\begin{tabular}{|l|c|}
\hline $\begin{array}{l}\text { Estudios del Centro de Investigaciones Sociológicas (CIS) que incluyen } \\
\text { preguntas sobre consumo político individual }\end{array}$ & TOTAL \\
\hline \multicolumn{2}{|c|}{ Estudio no 2450 Ciudadanía, Participación y Democracia (2002) } \\
\hline Boicotear o comprar productos por razones éticas, políticas o medioambientales & $6 \%$ \\
\hline Comprar productos por razones éticas, políticas o medioambientales & $12 \%$ \\
\hline \multicolumn{2}{|c|}{ Estudio no 2575 Ciudadanía y participación (2004) } \\
\hline $\begin{array}{l}\text { Boicotear o comprar ciertos productos por razones políticas o para favorecer el } \\
\text { medioambiente }\end{array}$ & $13 \%$ \\
\hline \multicolumn{2}{|c|}{ Estudio no 2588 Representación y Participación política (2005) } \\
\hline $\begin{array}{l}\text { Comprar o dejar de comprar ciertos productos deliberadamente por razones } \\
\text { políticas, éticas o medioambientales }\end{array}$ & $18 \%$ \\
\hline \multicolumn{2}{|c|}{ Estudio no 2606 Globalización y relaciones internacionales (2005) } \\
\hline $\begin{array}{l}\text { Boicotear o comprar ciertos productos por razones políticas, éticas o para favore- } \\
\text { cer el medio ambiente }\end{array}$ & $14 \%$ \\
\hline
\end{tabular}




\begin{tabular}{|l|c|}
\hline $\begin{array}{l}\text { Estudios del Centro de Investigaciones Sociológicas (CIS) que incluyen } \\
\text { preguntas sobre consumo político individual }\end{array}$ & TOTAL \\
\hline \multicolumn{2}{|c|}{ Estudio n' 2632 Ciudadanía y participación (2006) } \\
\hline $\begin{array}{l}\text { Boicotear o dejar de comprar ciertos productos por razones políticas, éticas o } \\
\text { para favorecer el medio ambiente }\end{array}$ & $14 \%$ \\
\hline $\begin{array}{l}\text { Comprar ciertos productos deliberadamente por razones políticas, éticas o para } \\
\text { favorecer el medio ambiente }\end{array}$ & $18 \%$ \\
\hline Recordaba alguna campaña de boicot a productos o empresas en España & $46 \%$ \\
\hline Se enteraron a través de los medios de comunicación & $84 \%$ \\
\hline \multicolumn{2}{|c|}{ Estudio no 2736 Internet y participación política (2010) } \\
\hline $\begin{array}{l}\text { Compró ciertos productos por razones políticas o para favorecer el medio am- } \\
\text { biente }\end{array}$ & $25 \%$ \\
\hline $\begin{array}{l}\text { Boicoteó o dejar de comprar ciertos productos por razones políticas, éticas o para } \\
\text { favorecer el medio ambiente }\end{array}$ & $20 \%$ \\
\hline \multicolumn{2}{|c|}{ Estudio no $\mathbf{2 8 6 0}$ Preferencias sobre los procesos de toma de decisiones políticas (2011) } \\
\hline $\begin{array}{l}\text { Boicoteado o dejado de comprar ciertos productos por razones políticas, éticas o } \\
\text { para favorecer el medio ambiente }\end{array}$ & $17 \%$ \\
\hline
\end{tabular}

Fuente: Elaboración propia a partir de los datos del CIS.

En España, el consumo político ha tenido una evolución progresiva en los últimos doce años. Según la tabla 1, se puede observar que el buycott es practicado por un mayor número de personas, como ocurre en el resto de los países. Así, en el año 2002, el 12\% de la ciudadanía lo había practicado, y, en 2010 la cifra se eleva al $25 \%$. Por otro lado, un $6 \%$ de la población afirmaba haber realizado boycott en 2002, mientras que, en 2010 el porcentaje se eleva al 20\%. Según estos datos, parece que la ciudadanía ha encontrado un nuevo espacio para expresar sus opiniones acerca de las políticas que llevan a cabo tanto los sucesivos gobiernos como las prácticas de las empresas o multinacionales.

En el marco del estudio de acción colectiva, la encuesta Actitudes, opiniones y comportamiento de la población andaluza en materia de consumo, realizada por el IESA - CSICE de Andalucía en 1998 aborda cuestiones de defensa de los consumidores. En ella se utiliza el concepto de "consumerismo" o la dimensión pública de la relación que mantiene la ciudadanía con la defensa de sus derechos como consumidores” (Ramírez, Navarro y Trujillo, 1999: 147; Moyano y Navarro, 1999; Navarro y Ramírez, 2000). Se analiza actitudes y comportamientos consumeristas de la población andaluza, perfil social del consumerismo, presencia social de las asociaciones de consumidores y el ciclo de protesta para ejercer sus derechos como consumidores. De las conclusiones del estudio, interesa saber que, el grupo que ha mostrado un nivel más alto en valores, conocimiento y comportamiento consumeristas responde al siguiente perfil: tener una ideología de izquierdas, identificarse con los valores postmaterialistas, ser joven o de edad madura, poseer un nivel alto de estudios y residir en hábitats urbanos, preferentemente en lugares donde hay OMIC (Ramírez, Navarro y Trujillo, 1999: 169). 
Otro estudio destacable es la Encuesta de hábitos alimentarios, seguridad e innovación alimentaria (Díaz, 2013) realizada a 1504 personas a nivel nacional, a través del sistema CATI, donde entre otros temas se analizan la participación social de los españoles en relación a la alimentación, etiquetado, confianza y riesgo alimentario. Así un 33\% hace un especial esfuerzo por comprar a empresas que apoyan causas sociales y un $66 \%$ firmaría para apoyar la protesta de asociaciones o grupos de consumidores.

\section{Consumidores políticos y estilos de vida}

Se puede decir que la mayoría de los estudios realizados hasta el momento se han interesado por el comportamiento individual y por conocer quiénes son y qué características tienen los consumidores que adoptan un estilo de vida en función de valores solidarios, de respeto a los derechos humanos o de protección al medioambiente (Stolle, Hooghe y Micheletti, 2003; Stolle y Micheletti, 2003; Forno y Ceccarini, 2004; Gould y Tobiasen 2004; Ferrer, 2004, 2006, 2010; Ferrer y Fraile, 2006, 2013).

Desde los primeros estudios clásicos sobre participación política (Lazarsfeld, et al 1948; Berelson, et al 1954; Milbrath y Goel, 1977; Barnes y Kaase, 1979) hasta la actualidad (Putnanm 2000; Norris, 2001, 2002, 2007; Ekman y Amna 2009, 2012) se ha demostrado que factores tales como los recursos individuales, las redes sociales, el grado de implicación con la política, sentimientos de eficacia o capacidad para influir sobre el sistema político y el contexto político e institucional son fuente de motivación para la participación de los ciudadanos.

A la hora de estudiar el componente político del consumo conviene tener en cuenta que está influido por una serie de variables de tal manera que es más probable allí donde se dan las siguientes características: ser mujer; joven; mayor nivel educativo; niveles de ingresos altos; pertenecer a asociaciones de nueva política; participación en manifestaciones o protestas; interés por la política; mayor cosmopolitismo y aceptación de la diversidad; mayor eficacia política interna y externa o sentir desconfianza hacia las instituciones (de acuerdo con la primera ola de la ESE realizada durante el período 2002-2003 y elaborada por Mariona Ferrer (2010: 246-254). En casi todos los trabajos se confirman las mismas relaciones entre variables, aunque cabe señalar que difieren en función del país y que en ninguno de ellos se han establecido comparaciones que permitan ponderar el efecto diferencial de estas variables en distintos contextos.

Kosss (2012:12) en su investigación a través de la ESS ha demostrado la importancia que tienen las oportunidades económicas y las instituciones políticas de los países europeos a la hora de influir en el comportamiento de consumo político de la ciudadanía. Por un lado, países económicamente prósperos permiten una mayor autonomía a los ciudadanos para decidir acerca de la adquisición o no de productos en el mercado. Por otro lado, una sociedad civil fuerte influye en una actitud activa hacia esta forma de participación. 
Según Newman y Bartels (2010) en EEUU es un fenómeno no muy estudiado y su trabajo ha sido el primero en analizar a nivel teórico, basándose en la perspectiva de la "lifestyle politics", y empírico, a través de la encuesta nacional Citizenship, Involvement, Democracy (CID) realizada por el Democracy and Civil Society at Georgetown University (Howard, Gibson, y Stolle, 2005) los factores que favorecen que a nivel individual los ciudadanos participen en esta nueva forma de participación extra-electoral. Newman y Bartels encuadran el estudio del consumo político en el marco más general de la participación política, a partir de una triple distinción: 1) si la participación es institucional o no institucional; 2) si la participación es individual o grupal y 3) el nivel de iniciativa ciudadana requerida para participar. Se basan en la "lifestyle politics" (teoría de la política del estilo de vida de Lance Bennet) para entender la dinámica causal a nivel individual del comportamiento del consumidor político. Concluyeron que es una forma de participación que se situaría en una dimensión institucional/no institucional (entre el voto y la protesta) en comparación con las tradicionales; el perfil del consumidor: nivel de educación alto, fuerte sentido del deber ciudadano, interés político, pertenencia a grupos, los más jóvenes y los que sienten desconfianza política y descontento sobre la situación actual de la vida (2010: 4-12).

Además, se han realizado estudios específicos que analizan el efecto de alguna de las variables que influyen en el consumo político. Ferrer y Fraile (2006) estudian los determinantes sociales del consumo político en Europa occidental a través de la ESE y demuestran, como pauta general, que los ciudadanos con mayores recursos, los de mayor nivel educativo, los estudiantes, empleados, los de mediana edad y los del sector servicios son más propensos a comportarse como consumidores políticos. En 2013 y a través también de la Encuesta Social Europea realizan una investigación sobre clase social y consumo político en los países de Europa occidental, los datos demostraron que la clase social influye y mucho en la probabilidad de participar en el consumo político (Ferrer y Fraile, 2013). Por otro lado, Llopis-Goig (2011: 93) realiza un estudio sobre consumo político y cosmopolitismo, tomando como base de datos el estudio $\mathrm{n}^{\circ} 2606$ del CIS, en donde uno de los principales hallazgos es que "el consumo político es una forma de acción a cuya génesis podría estar contribuyendo el cosmopolitismo". También Neilson (2006: 37-38) elabora un estudio a través de la ESE (2002-2003) en el que concluye que no sólo el capital social individual presenta una asociación positiva con el consumo político. El efecto que el capital social de una región podría ejercer se basaría en la relación mediada entre la participación en asociaciones, la confianza generalizada y los valores sociales. Este estudio muestra que el boicot está menos relacionado con el capital social individual que la compra de productos. Parece que ésta puede estar más influenciada por las relaciones interpersonales o el intercambio de información.

El descontento hacia instituciones de la democracia representativa incrementa el activismo vinculado a la red (Dalton, 2004; Putnamm, 2000; Dalton y Wattemberg, 2000) con la intención de influir en acontecimientos sociales, económicos y políticos. Son los más jóvenes los que han adoptado un nuevo rol "online” con el interés de influir en cierta medida en la realidad política activa. 
Así, en el contexto online, el estudio cuantitativo realizado a 1215 personas en el Reino Unido mediante una encuesta a través de Internet en el marco del proyecto CivicWeb encontró una relación positiva entre el consumo con conciencia social y la participación online y la participación cívica offline; sin embargo, esta relación desaparece con la participación política offline (Vard y Vreese, 2011: 410).

También es relevante reseñar algún trabajo realizado en torno a la acción colectiva de esta forma de participación. A este respecto, la investigación de Nelson, Rademacher y Paek (2007) se basa en los motivos para unirse al grupo internacional freecycle.org que se caracteriza por facilitar el libre intercambio de mercancías de segunda mano en la web. Entre sus intereses estaba el ver de qué forma se involucran en formas tradicionales y no tradicionales de participación política y tienden a practicar el consumo político (boycott y buycott) y explorar y revisar lo que significa ser cívica y políticamente comprometido en nuestra sociedad. Concluyen que las nuevas formas de consumo se pueden considerar como un tipo nuevo de compromiso cívico.

Otra investigación significativa es la de Francesca Forno (2007) que explora la estructura social de la movilización en Italia a través de la web para ilustrar algunas de las relaciones, los roles y las alianzas estratégicas que se han construido dentro de y en torno a las organizaciones facilitando la difusión del consumo político. Para realizar el rastreo de las webs relacionadas con este tipo de acción política se usó una herramienta desarrollada por Richard Rogers (2001) llamada ICrawler a través de la que se descubre la red anti-consumista italiana de los consumidores políticos. Sin lugar a dudas es un interesante método para identificar y visualizar esa red invisible de la comunicación interpersonal y organizacional (2007: 15).

Pero también se han realizado investigaciones cualitativas. La técnica de la entrevista en profundidad es la más usada entre los estudiosos de este fenómeno (Atkinson, 2013; Barnard, 2011). Así interesa destacar el trabajo de Lindén (2004) en el que elabora un estudio piloto con el objetivo de desarrollar una comprensión teórica de cómo los consumidores utilizan el etiquetado en función de sus valores y cómo determinan sus actitudes ante el consumo de alimentos o cuáles son las estrategias que usan los consumidores para influir sobre el mercado, sobre otros consumidores y sobre las políticas. Se utilizan las entrevistas en profundidad a 2 consumidores interesados, 5 vegetarianos y 2 veganos. También en la investigación de Connolly y Prothero (2008) se utilizaron entrevistas en profundidad a consumidores ecológicos. Para su selección se utilizó el método de muestreo estratégico de Mason, según el que se asigna un número a cada unidad de población y se extraen unidades de muestra de una tabla de números aleatorios. A través de su estudio se obtuvo una mayor comprensión de por qué la gente cree que como individuos puede llegar a solucionar un problema ambiental global, de hecho sentían que tenían la obligación de actuar en consecuencia.

De especial interés es el estudio sobre normalización de la protesta o la participación en manifestaciones de grupos heterogéneos de personas realizado por van Aeslt y Walgrave (2001). Utilizaron tres métodos de investigación de la acción colectiva: las encuestas de población, análisis de un evento de protesta y entrevistas 
con participantes en las manifestaciones. La conclusión de su investigación es que se ha producido una "normalización del manifestante con tal motivo deducen que se deben aceptar las recomendaciones de Tilly de que sería conveniente utilizar la investigación sobre acción colectiva como barómetro político (...). Sin embargo, la escasa representación de personas con nivel educativo y de ingresos bajo nos impide hablar de una verdadera democratización de la protesta en la calle” (íbid: 482). Y para finalizar, en el contexto "online” el estudio realizado por Janelle Ward (2008) presenta los resultados de una serie de entrevistas en profundidad con los encargados de la gestión de contenidos de páginas web de organizaciones juveniles con sede en Reino Unido. Parten del supuesto de que el consumo individual está ligado al concepto de ciudadanía, así examinan cómo las organizaciones utilizan Internet para dirigirse al joven ciudadano-consumidor. Una de las conclusiones a las que llega es que el consumo político es una nueva vía de acción de la ciudadanía.

\section{5. “Consumocracia” o el empoderamiento de los consumidores ante el mercado}

En la estructuración de este artículo se ha querido dar cuenta de los estudios más recientes relativos al consumo político. Según las teorías posmodernas que enlazan el mundo global con las nuevas formas de acción colectiva individualizada en el mercado, se abre ahora un nuevo espacio público en el que expresar y reivindicar cuestiones consideradas de justicia social. Los espacios participativos se amplían del mismo modo que nuevos actores políticos se incorporan a la vida democrática.

Así, al margen de la participación política tradicional, diariamente se toman decisiones motivadas como puede ser comprar café de comercio justo, o no comprar un alimento determinado de forma consciente, que tienen consecuencias políticas. De este modo se puede afirmar que es probable que el consumo de alimentos se haya politizado y cada vez más personas tomen decisiones cotidianas de consumo teniendo en cuenta cuestiones éticas como una mayor justicia social global, los derechos de las mujeres, la conciencia ambiental o los derechos de los animales.

$\mathrm{La}$ investigación futura debe identificar las conexiones entre el consumo con conciencia social y la ciudadanía democrática (Dulsrud y Jacobsen, 2007). En la actualidad, los debates teóricos giran en torno a la idea de que la búsqueda de un futuro más sostenible pasa por el "cambio de comportamiento" de un número importante de personas en la sociedad. Pese a las significativas conceptualizaciones entre las diferentes perspectivas teóricas, existe un consenso para determinar que el concepto de hábito es fundamental para poder entender los cambios en la conducta humana (Warde, y Southerton, 2012). Pero habría que señalar la necesidad de estudiar cualitativamente la autopercepción de la ciudadanía en relación a su hábito de consumo, para poder evidenciar la concordancia entre sus opiniones y sus acciones cívicas y políticas a través de las que incidir de manera consciente en el ejercicio democrático.

Por eso, parece conveniente partir de un modelo de ciudadanía activa en la que la participación democrática se materializa de un lado, a través de la "acción colec- 
tiva individualizada” (boycott y buycott), asumiendo la responsabilidad individual de convertir los hábitos de compra en hábitos políticos; y, de otro, como activistas en las diferentes organizaciones como cooperativas, ONG o movimientos sociales que actúan como intermediarios entre la política parlamentaria (o los gobiernos) y la política de la vida cotidiana (Siim, 2003: 5). Este tipo de participación significa el empoderamiento de los consumidores frente al mercado y al sistema político tradicional, carente este último de las competencias para hacer frente a los riesgos globales emergentes. De tal forma que ahora los consumidores políticos en tanto que actores sub-políticos (Micheletti, 2003; Sørensen, 2001, 2004; Tobiasen, 2004) utilizan un nuevo espacio fuera de la esfera tradicional (Beck, 1994; 1997; Micheletti, 2003). En cierto sentido, "los consumidores a través de la compra de determinados productos y no otros en el mercado están eligiendo el tipo de sociedad de la que quieren formar parte” (Brinkman, 2004:133).

A tal efecto se podría denominar "consumocracia” al empoderamiento de los consumidores, siendo que por medio de sus hábitos de compra reivindican reformas de las políticas públicas, buscando que sea la justicia social el eje articulador de las mismas. Hablar de consumocracia nos lleva a pensar la relación estrecha entre el consumo consciente diario de la compra y el ejercicio de ciudadanía en un espacio público como es el mercado. 


\section{Bibliografía}

Andersen, J.G. y M. Tobiasen (2001) Politisk forbrug og politiske forbrugere. Globalisering og politisk forbrug i hverdagslivet, (Political consumerism and Political Consumers. Globalization and Political Consumption in Everyday Life), Institut for Statskundskab, Aarhus.

Amnå, E., Ekman, T., y E. Almgren (2007) 'The end of a distinctive model of democracy? Country-diverse orientations among young adult Scandinavians" Scandinavian Political Studies, 30(1): 61-86.

Atkinson, L. (2013) "Buying In to Social Change: How Private Consumption Choices Engender Concern for the Collective" The ANNALS of the American Academy of Political and Social Science, 644: 191-206.

Barnard, A. (2011) '”Waving the banana' at capitalism: Political theater and social movement strategy among New York's 'freegan' dumpster divers” Ethnography, 12 (2): 419-444.

Barnes, S.y Kaase, M.et al (1979) Political Action: Mass Participation in Five Western Democracies London: Sage.

Beck, U. (1997) The Reinvention of Politics. Rethinking Modernity in the Global Social Order Cambridge: Polity Press.

Beck, U., Giddens, A y S. Lash (2001 [1997]) Modernización reflexiva. Política, tradición y estética en el orden social moderno Madrid: Alianza Universidad.

Bennett, W. L. (1998) "The uncivic culture: Communication, identity and the rise of lifestyle politics” Political Science and Politics, 31:741-61.

Bennet, L (2012) "The Personalization of Politics : Political Identity, Social Media, and Changing Patterns of Participation" The ANNALS of the American Academy of Political and Social Science, 644: 20-39.

Berelson, B. Lazarsfeld, P. y W. Macphee, (1954) Voting. A study of opinion formation in a presidential campaign, Chicago, The University of Chicago Press.

Berlin, (2011) "Sustainable Consumers and the State: Exploring How Citizens' Trust and Distrust in Institutions Spur Political Consumption” Journal of Environmental Policy \& Planning 13(3): 277-295.

Blanco, A (2006) "Responsabilidad social corporativa y desarrollo sostenible" Dirigir personas, 38: 36-40.

Boström, M. (2003) "Environmental organisations in new forms of political participation: Ecological modernisation and the making of voluntary rule", Environmental Values, 12:175-193.

Boström, M., Føllesdal, A., Klintman, M., Micheletti, M. y M. P. Sørensen (eds.), (2005) "Political Consumerism: Its Motivations, Power, and Conditions in the Nordic Countries and Elsewhere" [http://lib.icimod.org/record/12010/files/4048.PDF] (Ültima consulta: el el 25 de marzo de 2013).

Brinkman, J. (2004) "Looking at Consumer Behavior in a Moral Perspective" Journal of Business Ethics, 51:129-141. 
Clarke, B., Barnett, C., Clocke, P., A. Malpass (2007) "Globalising the consumer: Doing politics in an ethical register" Political Geography 26: 231-249.

Connolly, J. y A. Prothero (2008) "Green Consumption Life-politics, risk and contradictions” Journal of Consumer Culture, 8 (1): 117-145.

Dalton, R. y M. Wattenberg (2000) Parties without partisans. Political change in advanced industrial democracies Oxford: Oxford University Press.

Dalton, R. (2004) Democratic Challenges, Democratic Choices: The Erosion of Political Support in Advanced Industrial Democracies UK: Oxford University Press.

Dalton, R. J. (2008) "Citizenship norms and the expansion of political participation” Political Studies, 56(1): 76-98.

Díaz, C., (coord.) (2013) Hábitos alimentarios de los españoles Madrid: Ministerio de Agricultura, Alimentación y Medio Ambiente.

Dubuisson-Quellier, S. (2010) "From consumerism to the empowerment of consumers: the case of consumer-oriented movements in France" Sustainability, 2(7):1849-1868.

Echegaray, F. (2010) “¿Hacia la politización del consumo en Brasil? repensando el consumo ético desde la cultura política” Ambiente \&Sociedade, 13 (2): 383-400.

Ekman, J. y E. Amna (2009): "Political participation and civic engagement: Towards a new typology" Youth \& Society, YeS Working Paper (2).

Ekman, J. y E. Amna (2012): "Political Participation and Civic Engagement: Towars a New Tipology” Human Affairs, 22: 283-300.

Ferrer-Fons, M. (2004) "Cross-National Variation on Political Consumerism in Europe: Exploring the Impact of Micro-Level Determinants and its Political Dimension”, Paper presented at the ECPR Joint Sessions, Uppsala, Sweden.

Ferrer-Fons, M. y M. Fraile (2006) "Exploring the social determinants of political consumerism in Western Europe” Working Paper 57/2006, Departamento de Ciencia Política, Universidad Autónoma de Madrid.

Ferrer-Fons, M. (2010) "El estudio del consumo político en Europa: ¿una forma de acción política de la ciudadanía del siglo XXI?” en Torcal, M. La ciudadanía europea en el siglo XXI. Estudio comparado de sus actitudes, opinión pública y comportamientos políticos Madrid: CIS, 237-264.

Ferrer-Fons, M y M. Fraile (2013) "Political Consumerism and the Decline of Class Politics in Western Europe" International Journal of Comparative Sociology 16. Doi:10.1177/0020715213516476

Forno, F., y L. Ceccarini (2006) "From the Street to the Shops: The Rise of New Forms of Political Actions in Italy" South European Society and Politics, 11(2): 197-222.

Forno, F. (2007) “Studying Political Consumerism on the Web”, Paper prepared for the 4th ECPR General Conference, Pisa (Italy), 6-8 September, 2007 Section: "Emerging Patterns of Collective Action" Panel: PP1742 "The use of ICTs for innovative forms of participation".

Gould, A. y M. Tobiasen (2004) "Who are these political consumers anyway? Survey evidence from Denmark", en Micheletti, M, Follesdal, A. y D. Stolle 
(eds.) Politics, and Markets. Exploring political consumerism past and present New Brunswick: Transaction Press.

Gotlieb, M. y C. Wells (2012) "From Concerned Shopper to Dutiful Citizen: Implications of Individual and Collective Orientations toward Political Consumerism” The ANNALS of the American Academy of Political and Social Science, 644 (1): 207-219.

Halkier, B. (2004) "Handeling Food-related Risks: Political Agency and Governmentality” en Lien, M. y B. Nerlich, (eds.) The Politics of Food Oxford: Berg.

Hertz, N. (2001) "Better to Shop Than to Vote?” Business Ethics A European Review 10:190-193.

Holzer, B. y M. P. Sørensen (2003) "Rethinking Subpolitics: Beyond the 'Iron Cage' of Modern Politics?” Theory, Culture \& Society 20(2): 79-102.

Hooghe, M y M. Stolle (2012) "How to reach Members of Parliament? Citizens and Members of Parliament on the Effectiveness of Political Participation Repertoires” Parliamentary Affairs 66 (1).

Howard, M., Gibson, J y D. Stolle (2005) The U.S. Citizenship, Involvement, Democracy Survey Washington, DC: Georgetown University Center for Democracy and Civil Society.

Inglehart, R., (1997) Modernization and postmodernization: Cultural, economic, and political changein 43 societies Princeton, NJ.: Princeton University Press.

Jacobsen, E. y Dulsrud, A. (2007) "Will consumers save the world? The framing of political consumerism” Journal of Agricultural and Environmental Ethics 20: 469-482.

Johnston, J. (2008) "The citizen-consumer hybrid: ideological tensions and the case of Whole Foods Market” Theory and Society, 37(3): 229-270.

Kelley, F. (1899/2008) "Metas y principios de la Liga de Consumidores”, Athenea Digital 14: 317-327,

[http://psicologiasocial.uab.es/athenea/index.php/atheneaDigital/article/view/585]

(ültima visita: el 17 de mayo de 2013).

Klimtan, M. y M. Boström (2004) "Framings of Science and Ideology: Organic Food Labelling in the US and Sweden” Environmental Politics 13: 612-633.

Koos, S. (2012) "What drives political consumption in Europe? A multi-level analysis on individual characteristics, opportunity structures and globalization" Acta Sociologica, 55(1): 37-57.

Lazarsfled, P., Berelson, B. y H. Gaudet (1948) The People's Choice: How the Voter Makes Up His Mind in a Presidential Campaign New York: Columbia University Press.

Lindén, A., (2004) "Private Food Strategies and Political Consumerism" en Boström, M., Føllesdal,A. Klintman,M., Micheletti, M. y Sørensen, M. (eds) Political Consumerism: Its motivations, power, and conditions in the Nordic countries and elsewhere. Proceedings from the 2nd International Seminar on Political Consumerism, Oslo August 26-29: 203-224. 
Llopis-Goig, R. (2011) "Consumo político y cosmopolitismo. Un estudio de participación política postconvencional en Espana” REIS 135: 89-106.

Marien, S., Hooghe, M., y E. Quintelier, (2010) "Inequalities in noninstitutionalised forms of political participation: A multi-level analysis of 25 countries” Political Studies, 58(1): 187-213.

McGregor, S., (2002) "Consumer Citizenship: A Pathway to Sustainable Development?" Presented at the International Conference on Developing Consumer Citizenship, April 2002, Hamar, Norway.

[http://www.consultmcgregor.com/documents/keynotes/norway_keynote.pdf.] (Última consulta: 9 de abril de 2013).

Micheletti, M., (2002) "Consumer Choice as Political Participation" Statsvetenskaplig Tidskrift 105 (3): 218-234.

Micheletti, M., (2003) Political Virtue and Shopping. Individuals, Consumerism, and Collective Action New York: Palgrave.

Micheletti, M., Follesdal, A. y D. Stolle (2003) Politics, Products, and Markets. Exploring Political Consumerism Past and Present New Brunswick, NJ.:Transaction Publishers.

Micheletti, M. y D. Stolle (2005) "Swedish political consumers: Who they are and why they use the market as an arena for politics" en Boström, A. F. M, Klintman, M., Micheletti, M. y M. P. Sørensen, (eds) Political Consumerism: Its Motivations, Power, and Conditions in the Nordic Countries and Elsewhere (Oslo: TemaNord): 145-164.

Micheletti, M., (2010) Political virtue and shopping: Individuals, consumerism, and collective action, 2nd ed. New York NY: Palgrave.

Micheletti, M. y D. Stolle (2012) "Sustainable Citizenship and the New Politics of Consumption" The ANNALS of the American Academy of Political and Social Science 644 (1): 88-120.

Micheletti, M., Stolle, D. y D. Berlin (2012) "Habits of Sustainable Citizenship: The Example of Political”.

[http://www.sustainablecitizenship.com/pdf/12_09_micheletti-stolle-berlin.pdf] (Úlitma consulta: 9 de abril).

Milbrath, L. y Goel (1977) Political participation. How and why people get involved in politics Chicago: Rand McNally.

Moyano, E., y C. J. Navarro (1999) El movimiento de los consumidores en Andalucía, Sevilla: Consejería de Trabajo e Industria de la Junta de Andalucía.

Navarro, C. J., y A. Ramírez (2000) "La defensa individual de intereses públicos. Propuesta metodológica para el caso de los consumidores" Estudios sobre Consumo, 55: 31-69, Instituto Nacional de Consumo.

Neilson, L. A. y P. Paxton (2010) "Social capital and political consumerism: A multilevel analysis” Social Problems, 57(1): 5-24.

Nelson, M., Rademacher, M. y H. Paek, (2007)“Downshifting consumer upshifting citizen? An examination of a local freecycle community" The Annals of the American Academy of Political and Social Science 611: 141-56. 
Newman, B. J., y B. L. Bartels (2010) "Politics at the checkout line: Explaining political consumerism in the United States" Political Research Quarterly, XX (X): 1-15.

Norris, P. (2001) Digital divide? Civic engagement, information poverty and the internet worldwide Cambridge: Cambridge University Press.

Norris, P. (2002) La participación ciudadana: México desde una perspectiva comparativa Cambridge: University of Harvard.

Norris, P. (2002) Democratic Phoenix. Reinventing Political Activism Cambridge: Cambridge University Press.

Parry, G., Moyser, G., y N. Day (1992) Political participation and democracy in Britain Cambridge: Cambridge University Press.

Putnam, R. (2000) Bowline alone: The collapse and revival of American community New York: Simon and Schuster.

Ramírez, A., Navarro, C., y M. Trujillo, (1999) "Consumerismo y movimiento de los consumidores Valores, actitudes y comportamiento consumeristas de la población andaluza“ REIS 99 (02): 145-176.

Rask Jensen, H. (2001) "A Frame of Reference for Analyzing Political Consumption" Paper for the International Seminar on Political Consumerism, Stockholm, May/June.

Shah, D. V., McLeod, D. M., Friedland, L., y M. R. Nelson (2007) "The politics of consumption/the consumption of politics" Annals of the American Academy of Political and Social Science, 611(1): 6-15.

Siim, B. (2003) "Gender Equality, Citizenship and Globalisation", this paper is a revised version of the keynote speech "How to Achieve Gender Equality" for the COST A 13Conference Social Policy, Marginalisation and Citizenship, Aalborg University, Denmark, 2-4. November, 2001 to be published in J.G. Andersen, AM. Guillemard, Per H. Jensen y B. Pfau-Effinger (eds), Social Politics, Social Integration and Citizenship by Polity Press, book series.

[http://www.monnet-centre.uni-bremen.de/GenderGlobNet/Paper/PaperSiim1.pdf.]

(Última consulta: el 20 de marzo de 2013).

Stolle, D, Hooghe, M. y M. Micheletti (2005) "Politics in the supermarket: Political consumerism as a form of political participation" International Political Science Review 26: 245-69.

Stolle, D. y M. Micheletti (2005) "What motivates Political Consumers?. For Online Forum: Politisierter Konsum - konsumierte Politik", first draft for the Special Issue on "The Underestimated Consumer-Power - Prospects for the New Consumer Movement” in "Forschungsjournal Neue Soziale Bewegungen” (No. 4, 2005)

[http://www.politik-konsum.de/pdf/fnsb_stolle_micheletti.pdf.] (Última consulta: el 28 de marzo de 2013).

Stolle, D., y M. Hooghe, (2011) "Shifting inequalities: Patterns of exclusion and inclusion in emerging forms of political participation” European Societies, 13(1): 119-142. 
Strømsnes, K., (2009) "Political consumerism: A Substitute for or Supplement to Conventional Political Participation?” Journal of Civil Society 5(3): 3030-3031.

Tobiasen, M., (2004) Political Consumers in Denmark Paper for the ECPR Joint Sessions, Uppsala, Sweden

[http://lib.icimod.org/record/12010/files/4048.PDF] (Última consulta: 25 de mayo de 2013).

Todt, O. y M. I González (2006) “Del gobierno a la gobernanza” Isegoría 34: 209224.

Van Deth, J. (2001) "Studying Political Participation: Towards a Theory of Everything? Introductory paper prepared for delivery at the Joint Sessions of Workshops of the European Consortium for Political Research. Workshop "Electronic Democracy: Mobilisation, Organisation and Participation via new ICTs". Grenoble.

Van Aelst, y S. Walgrave (2001) "Who is that (wo)man in the street? From the normalisation of protest to the normalisation of the protester" European Journal of Political Research 39: 461-486.

Van Laer, J. y P. Van Aelst (2009) "Cyber-Protest and Civil Society: the Internet and Action Repertoires of Social Movements” en Jewkes, Y. y Y. Majid (eds.) Handbook on Internet Crime Portland: Universia Press.

Verba, S., Schlozman, K. y H. BRADY (1995) Voice and equality: Civic voluntarism in American politics Cambridge: Cambridge University Press.

Ward, J. (2008) "The online citizen-consumer: Addressing young people's political consumption through technology” Journal of Youth Studies, 11(5): 513-526.

War, J. y C. de Vreese, (2011) "Political consumerism, young citizens and the Internet” Media, Culture \& Society 33(3): 399-413.

Warde, A. Y D. Southerton, (eds.) (2012) "The Habits of Consumption" COLLeGIUM of Studies Across Disciplines in the Humanities and Social Sciences, Volume 12.

Webster, Jr., (1975) "Determining the characteristics of the socially conscious consumer" Journal of Consumer Research 2:188-96.

Young, I. (1990) Justice and the Politics of Difference Princeton N.J.: Princetong University Press.

Young, I., (1994) "Gender as Seriality: Thinking about Women as a Social Collective” Signs: Journal of Women in Culture and Society 19: 713-38. 